\title{
Corpo Peritoneal Livre Gigante: Caso Clínico e Revisão da Literatura
}

\section{Giant Peritoneal Loose Body: Case Report and Review of Literature}

Rodrigo OOM $₫ 1$, Cátia CUNHA 2 , Vítor Moura GUEDES ${ }^{2}$, Luís Palma FÉRIA², Rui MAIO²

Acta Med Port 2018 May;31(5):272-276 - https://doi.org/10.20344/amp.9177

\section{RESUMO}

Os corpos peritoneais livres são habitualmente diagnosticados de forma incidental. Apenas alguns casos estão documentados na literatura. A sua fisiopatologia não é totalmente conhecida, estando eventualmente relacionada com a torção e separação dos apêndices epiplóicos. É descrito o caso de um homem com 64 anos de idade, com uma lesão sólida esférica de $6 \mathrm{~cm}$ de diâmetro na cavidade pélvica, identificada incidentalmente por tomografia computorizada. Foi realizada laparotomia exploradora, identificando-se uma lesão livre, de morfologia ovoide e esbranquiçada. O exame histológico mostrou uma massa de esteatonecrose calcificada e capsulada. Foi feita uma revisão dos casos de corpos peritoneais livres gigantes descritos na literatura. A excisão cirúrgica é recomendada quando o diagnóstico é duvidoso ou quando as lesões são sintomáticas. Os médicos devem ter conhecimento desta entidade, para poderem estabelecer um diagnóstico e plano de tratamento corretos.

Palavras-chave: Calcinose; Doenças Peritoneais

\section{ABSTRACT}

Peritoneal loose bodies are usually diagnosed incidentally. Only a few cases are documented in the literature. The pathophysiology of this condition is not fully known and its origin may possibly be related to the twisting and separation of epiploic appendages. The authors describe the case of a patient with a spherical solid lesion $6 \mathrm{~cm}$ in diameter, identified incidentally in the pelvic cavity of a 64 year old man. The patient underwent laparotomy and a free ovoid shaped white body was identified. Histological examination described a calcified and encapsulated mass with a steato-necrosis core. A review of all cases of giant peritoneal loose bodies described in the literature is included. Surgical excision is recommended when the giant peritoneal loose body diagnosis is uncertain or when they are symptomatic. Doctors should be aware of its existence in order to establish a proper diagnosis and treatment plan.

Keywords: Calcinosis; Peritoneal Diseases

\section{INTRODUÇÃO}

A incidência dos corpos peritoneais livres (CPL) não é clara na literatura médica. Estão descritos apenas alguns casos com mais de $5 \mathrm{~cm}$, considerados corpos peritoneais livres gigantes (CPLG). ${ }^{1}$

O diagnóstico dos CPL habitualmente não é imediato. Os CPL são frequentemente assintomáticos e identificados em autópsias ou cirurgias, mas o seu crescimento pode originar sintomas, implicando um desafio para o diagnóstico e terapêutica. ${ }^{2-7}$

O objectivo deste trabalho é apresentar um caso clínico de um doente com uma massa abdominal rara, de comportamento benigno, tratada por abordagem cirúrgica simples e diagnosticada como um CPL. Foi realizada ainda uma revisão da literatura dos casos desta entidade.

Os dados do presente caso clínico foram recolhidos do registo eletrónico hospitalar do doente em estudo. A revisão da literatura foi realizada pela pesquisa do termo giant peritoneal loose body no PubMed, tendo sido avaliados todos os artigos desta temática descritos como casos clínicos ou em revisões prévias.

\section{CASO CLÍNICO}

Doente do sexo masculino, raça caucasiana, com 64 anos de idade, referenciado à consulta de Cirurgia Geral por massa pélvica de etiologia desconhecida. O doente apresentava antecedentes de hipertensão arterial, diabetes mellitus não insulino-dependente, bronquite crónica, gota, dislipidémia, obesidade e artroplastia do joelho.

Estava assintomático quando recorreu à consulta de Medicina Geral e Familiar para a realização de análises clínicas de rotina. Nestas, detetou-se um valor de antigénio prostático específico (PSA) elevado, sem outras alterações analíticas relevantes. Realizou ecografia prostática trans-rectal que, para além de descrever um aumento ligeiro do volume da próstata, revelou uma massa esférica em contacto com a bexiga. Para estudo desta massa, fez uma tomografia computadorizada (TC) abdomino-pélvica, que identificou uma lesão nodular sólida com calcificação nodular central, com pequeno componente de gordura na sua vertente anterior esquerda e com diâmetro máximo de $6 \mathrm{~cm}$. A lesão contactava a parede lateral da bexiga, mas aparentemente era independente desta. A bexiga apresentava paredes de contornos regulares e com conteúdo homogéneo (Fig. 1).

No contexto da lesão pélvica de etiologia desconhecida, realizou-se uma laparotomia exploradora. Esta identificou uma massa livre na cavidade pélvica, esbranquiçada, com forma ovóide e contornos regulares (Fig. 2). A exploração

1. Serviço de Cirurgia Geral. Instituto Português de Oncologia de Lisboa Francisco Gentil. Lisboa. Portugal.

2. Serviço de Cirurgia Geral. Hospital Beatriz Ângelo. Loures. Portugal.

$\triangle$ Autor correspondente: Rodrigo Oom. rodrigo.oom@gmail.com

Recebido: 08 de maio de 2017 - Aceite: 29 de novembro de 2017 | Copyright @ Ordem dos Médicos 2018 


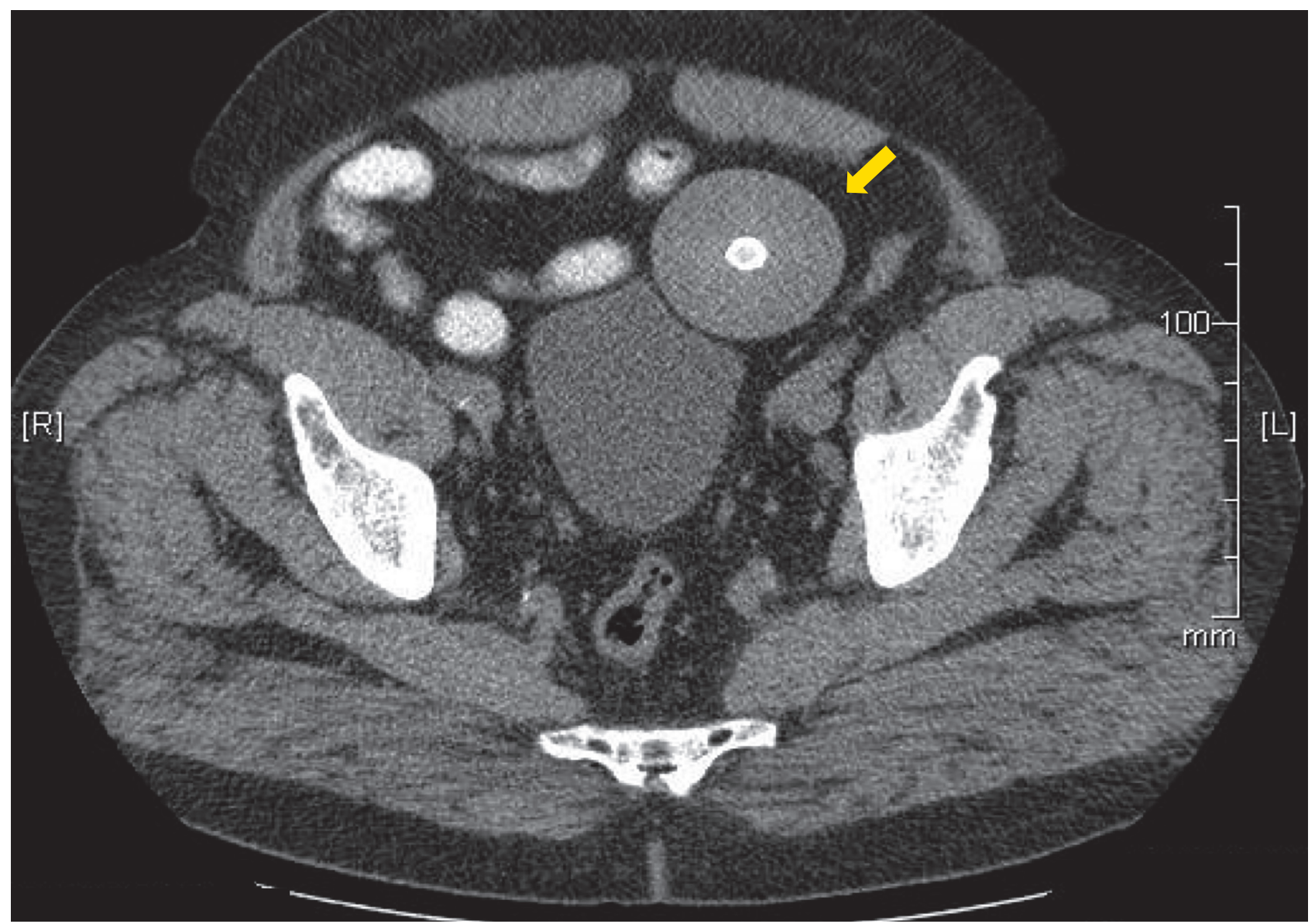

Figura 1 - Tomografia computorizada evidencia imagem esférica na cavidade pélvica em contacto com a bexiga (seta)
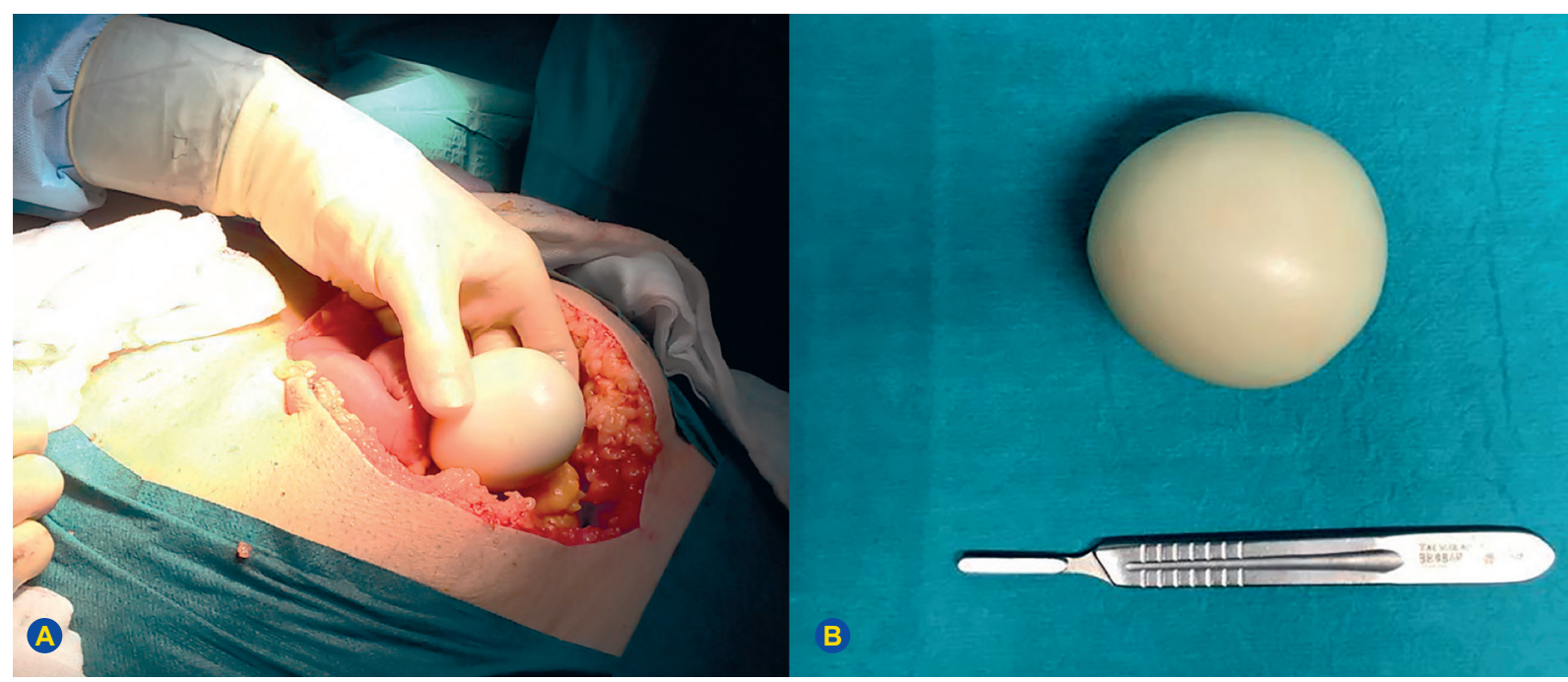

Figura 2 - Laparotomia infra-umbilical com excisão de estrutura esférica esbranquiçada livre na cavidade pélvica (A); Peça operatória (B)

da cavidade abdominal não identificou outras alterações, líquido livre ou lesões peritoneais, pelo que se procedeu à remoção da lesão. O pós-operatório decorreu sem intercorrências, e o doente teve alta ao terceiro dia.

A avaliação macroscópica da peça operatória identificou uma formação ovóide com $101 \mathrm{~g} \mathrm{e} 6$ × 4 ×6 cm, com superfície lisa e branca e que em secção, apresentava uma superfície laminada, elástica e centrada por formação cal- cificada com $1 \mathrm{~cm}$ de maior eixo (Fig 3). O exame microscópico revelou uma lesão centrada por calcificação com padrão alveolar correspondendo a esteatonecrose, envolvida por tecido acelular, laminar e disposto de forma concêntrica. Estes aspectos morfológicos eram sugestivos de necrose de apêndice epiplóico com posterior calcificação, deposição e precipitação de material proteináceo. 


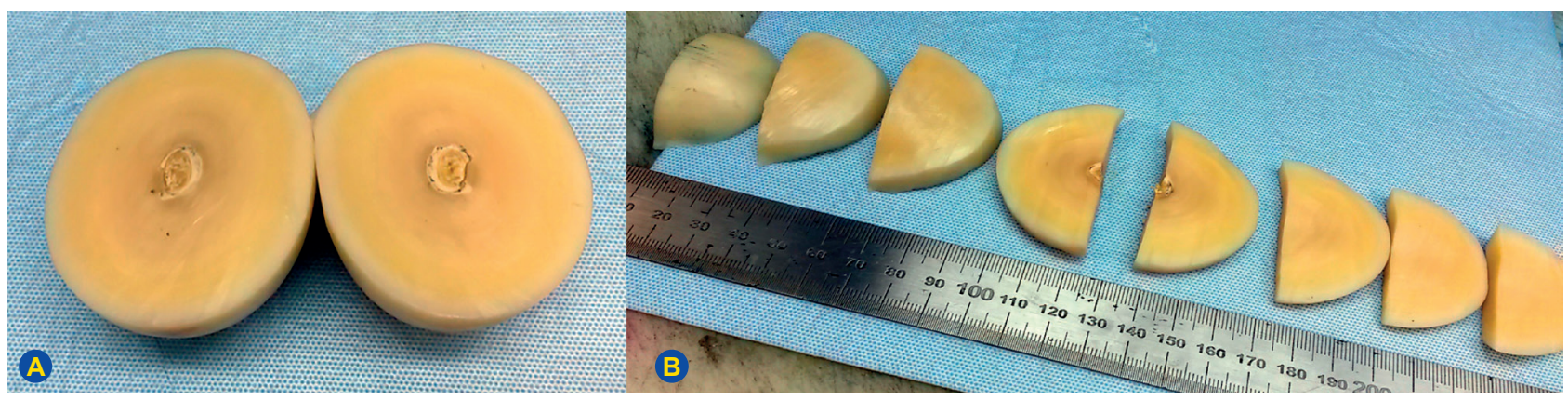

Figura 3 - Avaliação macroscópica de peça operatória (A, B)

\section{DISCUSSÃO}

Os CPL são normalmente diagnosticados incidentalmente em exame de imagens ou durante cirurgias ou autópsias. ${ }^{6}$ Este caso de CPLG é, do nosso conhecimento, o único descrito na literatura médica portuguesa.

A sua patogénese não é completamente conhecida. Alguns autores defendem diferentes origens entre os quais, os apêndices epiplóicos, grande epíploon, ${ }^{7}$ tecido pancreático ${ }^{5}$ ou órgãos anexiais. ${ }^{8} \mathrm{~A}$ hipótese mais frequente é ter origem num apêndice epiplóico, o qual sofre um processo sequencial: 1) torção do apêndice epiplóico; 2) isquémia; 3) saponificação; 4) calcificação; 5) separação do cólon devido à atrofia do pedículo e formação do corpo peritoneal livre ${ }^{7}$. Este fenómeno de torção dos apêndices epiplóicos foi já descrito em 1917 por Harrigan. ${ }^{2}$

Estas entidades são geralmente semelhantes entre elas: estruturas brancas de forma ovóide, margens regulares, consistência de borracha e em alguns casos mais calcificadas, ${ }^{9}$ tal como o caso descrito (Fig. 3). Alguns autores sugerem que estes corpos peritoneais vão gradualmente absorvendo líquido peritoneal e aumentando progressivamente de dimensões. A maioria tem dimensões de 0,5 a 2,5 $\mathrm{cm}$ de maior eixo mas quando o seu diâmetro ultrapassa os $5 \mathrm{~cm}$, são considerados CPLG, tal como o caso descrito. A maioria dos CPLG ocorre em doentes do sexo masculino entre a terceira e sétima década de vida e a lesão de maiores dimensões descrita na literatura tem $20 \mathrm{~cm}^{10}$ (Tabela 1). O tempo de crescimento destas entidades e os factores condicionantes para o seu crescimento também não são claros. Alguns autores descrevem crescimento de $2,5 \mathrm{~cm}$ em cinco anos ${ }^{7}$ enquanto outros não encontraram alteração de dimensões ao longo de pelo menos três anos. ${ }^{11}$

Tabela 1 - Casos de corpos peritoneais livres descritos na literatura

\begin{tabular}{|c|c|c|c|c|c|c|c|}
\hline Autor & Ano & Sexo & Idade & Sintomas & $\begin{array}{l}\text { Exame } \\
\text { imagem }\end{array}$ & $\begin{array}{l}\text { Dimensão } \\
\text { (maior eixo em cm) }\end{array}$ & Terapêutica \\
\hline Shepherd et $a /^{13}$ & 1951 & $M$ & 79 & Retenção urinária & $\mathrm{Rx}$ & 7,0 & Laparotomia \\
\hline Bhandarwar et a/15 & 1996 & $M$ & 65 & Retenção urinária & Não realizado & 9,0 & Laparotomia \\
\hline Takada et a/ & 1998 & M & 79 & Assintomático & TC e RM & 7,0 & Laparotomia \\
\hline Nomura et $a l^{16}$ & 2003 & $M$ & 63 & Assintomático & TC e RM & 5,0 & Laparoscopia \\
\hline Ghosh et all7 & 2006 & M & 63 & Oclusão intestinal & TC & 5,8 & Laparoscopia \\
\hline Mohri et al ${ }^{1}$ & 2007 & $M$ & 73 & Dor abdominal & $\mathrm{TC}$ & 9,5 & Laparotomia \\
\hline Hedawoo and Wagh et al ${ }^{18}$ & 2010 & M & 65 & Obstipação & $\mathrm{TC}$ & 9,5 & Laparotomia \\
\hline Sewkani et $a l^{14}$ & 2011 & $M$ & 64 & Oclusão intestinal & $\mathrm{Rx}$ & 7,0 & Laparotomia \\
\hline Kim et $a /^{19}$ & 2013 & M & 50 & Assintomático & TC & 7,5 & Laparoscopia \\
\hline Rajbhandari et $a^{20}$ & 2013 & M & 67 & $\begin{array}{l}\text { Sintomas } \\
\text { neurológicos } \\
\text { membro inferior }\end{array}$ & Eco e TC & 5,0 & Laparoscopia \\
\hline Sahadev et $a^{R^{1}}$ & 2014 & M & 52 & Dor abdominal & Eco e TC & 7,0 & Laparoscopia \\
\hline Makineni et $a^{22}$ & 2014 & M & 52 & Dor abdominal & TC & 6,0 & Laparotomia \\
\hline Rubinkiewicz et al ${ }^{10}$ & 2014 & $\mathrm{~F}$ & 78 & Oclusão intestinal & Rx e Eco & 20,0 & Laparotomia \\
\hline Suganuma et $a^{23}$ & 2015 & $\mathrm{~F}$ & 35 & Assintomático & Eco e RM & 7,5 & Laparoscopia \\
\hline Zhang et $a^{24}$ & 2015 & M & 51 & Assintomático & Eco e TC & 5,0 & Laparoscopia \\
\hline Elsner et $a^{25}$ & 2016 & M & 52 & Proctite & TC & 5,2 & Laparoscopia \\
\hline Rosic et a ${ }^{26}$ & 2016 & $M$ & 72 & Retenção urinária & TC & 6,6 & Laparoscopia \\
\hline Matsubara et $a^{27}$ & 2017 & M & 70 & Retenção urinária & TC & 5,8 & Laparoscopia \\
\hline Huang et $a^{28}$ & 2017 & M & 79 & Retenção urinária & Eco e TC & 10,4 & Laparotomia \\
\hline
\end{tabular}

Rx: radiografia; TC: tomografia computorizada; RM: ressonância magnética; Eco: ecografia 
Normalmente os CPL são assintomáticos, contudo, podem estar associados a sintomas de dor abdominal, ${ }^{7,12}$ retenção urinária ${ }^{13}$ ou oclusão intestinal. ${ }^{14} \mathrm{~A}$ sua localização mais frequente é na cavidade pélvica pela sua posição a favor da gravidade.

O diagnóstico diferencial é feito com doenças benignas (teratoma, fibroma, leiomioma uterinos calcificados, litíase urinária ou vesicular), malignas (cancro do ovário, colo-rectal ou metástases peritoneais) e outras entidades como corpos estranhos ou adenopatias. Os exames imagiológicos, como a ecografia, a tomografia computorizada ou a ressonância magnética, são importantes para o seu diagnóstico sendo a imagem mais frequente dos CPL uma estrutura de forma oval, bem delimitada e com centro calcificado rodeado por tecido fibroso. A realização de exame de imagem com o doente em diferentes posições pode facilitar o diagnóstico do CPL pela sua mobilidade. ${ }^{6}$

Em apenas um caso revisto na literatura, o diagnóstico foi realizado corretamente antes da cirurgia ${ }^{1}$ provavelmente devido à raridade destas lesões e pelo facto dos médicos não estarem familiarizados com esta entidade. A excisão cirúrgica e o exame patológico subsequente são o método de eleição para estabelecer o diagnóstico definitivo.

Nos doentes assintomáticos e com diagnóstico imagiológico inequívoco, é possível optar por uma abordagem conservadora de vigilância. ${ }^{11}$ Está indicado o tratamento cirúrgico quando estas lesões são sintomáticas ou quando o diagnóstico não está esclarecido. A abordagem cirúrgica que tem sido mais utilizada é a laparotomia mas a via laparoscópica é uma opção, sendo mais fácil de selecionar se o diagnóstico for considerado no pré-operatório, o que é pouco frequente. ${ }^{12}$ Retrospectivamente, no caso descrito, a via laparoscópica poderia ter sido realizada, mas o diagnóstico de CPL não foi considerado no pré-operatório.

\section{CONCLUSÃO}

Os CPLG são entidades raras e diagnosticados incidentalmente na maioria dos casos.

O seu diagnóstico nem sempre é fácil de realizar através de métodos complementares de diagnóstico, sendo a exploração cirúrgica recomendada quando o diagnóstico é duvidoso ou na presença de sintomas. A exploração por via laparoscópica deve ser a abordagem inicial, uma vez que a identificação de uma massa calcificada livre na cavidade abdominal sugere este diagnóstico, que deve ser confirmado histologicamente.

Este caso demonstra que o conhecimento da patologia é importante para que o plano de tratamento seja o mais apropriado, pelo que a apresentação e discussão de um caso clínico exemplar poder ser útil para médicos de várias especialidades, como a Cirurgia Geral, a Urologia, a Imagiologia ou mesmo para os médicos generalistas.

\section{PROTEÇÃO DE PESSOAS E ANIMAIS}

Os autores declaram que os procedimentos seguidos estavam de acordo com os regulamentos estabelecidos pelos responsáveis da Comissão de Investigação Clínica e Ética e de acordo com a Declaração de Helsínquia da Associação Médica Mundial.

\section{CONFIDENCIALIDADE DOS DADOS}

Os autores declaram ter seguido os protocolos do seu centro de trabalho acerca da publicação de dados.

\section{CONSENTIMENTO DO DOENTE}

Obtido.

\section{CONFLITOS DE INTERESSE}

Os autores declaram não terem qualquer conflito de interesse relativamente ao presente artigo.

\section{FONTES DE FINANCIAMENTO}

Não existiram subsídios ou bolsas que tenham contribuído para a realização do trabalho.

obstruction - report of a case. Pol Przegl Chir. 2014;86.7:341-4

11. Gayer G, Petrovitch I. CT diagnosis of a large peritoneal loose body: a case report and review of the literature. Br J Radiol. 2011;84:83-5.

12. Murat FJ, Gettman MT. Free-floating organized fat necrosis: rare presentation of pelvic mass managed with laparoscopic techniques. Urology. 2004;63.1:176-7.

13. Shepherd JA. Peritoneal loose body causing acute retention of urine. $\mathrm{Br}$ J Surg. 1951;39:185-7.

14. Sewkani A, Jain A, Maudar K, Varshney S. Boiled egg' in the peritoneal cavity-a giant peritoneal loose body in a 64-year-old man: a case report. J Med Case Rep. 2011;5:297.

15. Bhandarwar AH, Desai VV, Gajbhiye RN, Deshraj BP. Acute retention of urine due to a loose peritoneal body. Br J Urol. 1996;78:951-2.

16. Nomura H, Hata F, Yasoshima T, Kuwahara S, Naohara T, Nishimori H, et al. Giant peritoneal loose body in the pelvic cavity: report of a case. Surg Today. 2003;33:791-3.

17. Ghosh P, Strong C, Naugler W, Haghighi P, Carethers JM. Peritoneal mice implicated in intestinal obstruction: report of a case and review of the literature. J Clin Gastroenterol. 2006;40:427-30.

18. Hedawoo JB, Wagh A. Giant peritoneal loose body in a patient with haemorrhoids. Trop Gastroenterol. 2010;31:132-3. 
19. Kim HS, Sung JY, Park WS, Kim YW. A giant peritoneal loose body. Korean J Pathol. 2013;47:378-82.

20. Rajbhandari M, Karmacharya A, Shrestha S. Pathological diagnosis of peritoneal loose body: a case report. J Pathol Nepal. 2013;3: 512-4.

21. Sahadev R, Nagappa PK. A case of peritoneal free floating calcified fibromyoma. J Clin Diagn Res. 2014;8: ND03-4.

22. Makineni $\mathrm{H}$, Thejeswi P, Prabhu S, Bhat RR. Giant peritoneal loose body: a case report and review of literature. J Clin Diagn Res. 2014;8:187-8.

23. Suganuma I, Mori T, Takahara T, Torii H, Fujishiro M, Kihira T, et al. Autoamputation of a pedunculated, subserosal uterine leiomyoma presenting as a giant peritoneal loose body. Arch Gynecol Obstet. 2015;291:951-3.

24. Zhang H, Ling YZ, Cui MM, Xia ZX, Feng Y, Chen CS. Giant peritoneal loose body in the pelvic cavity confirmed by laparoscopic exploration: a case report and review of the literature. World J Surg Oncol. 2015;13:118.

25. Elsner A, Walensi M, Fuenfschilling M, Rosenberg R, Mechera $R$. Symptomatic giant peritoneal loose body in the pelvic cavity: A case report. Int J Surg Case Rep. 2016;21:32-5.

26. Rosic T, Khumalo V, Nethathe DG. A pelvic "loose" body tumour causing urinary symptoms in a 73-year-old man. S Afr J Surg. 2016;54:53-4.

27. Matsubara K, Takakura $\mathrm{Y}$, Urushihara T, Nishisaka T, Itamoto T. Laparoscopic extraction of a giant peritoneal loose body: case report and review of literature. Int J Surg Case Rep. 2017;39:188-91.

28. Huang Q, Cao A, Ma J, Wang Z, Dong J. Two giant peritoneal loose bodies were simultaneously found in one patient: a case report and review of the literature. Int J Surg Case Rep. 2017;36:74-7.

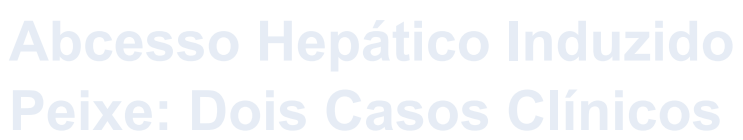

José Eduardo MATEUSW1, Carlos SILVA1, Sofia BEIRÃO22, Jorge PIMENTEL
Acta IMed Port 2018 May;31(5):276-279 " https://doi.org/10.20344/amo.9662

ABSTRACT

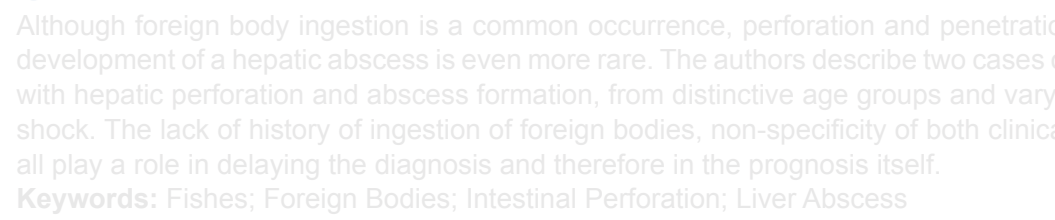

RESUMO

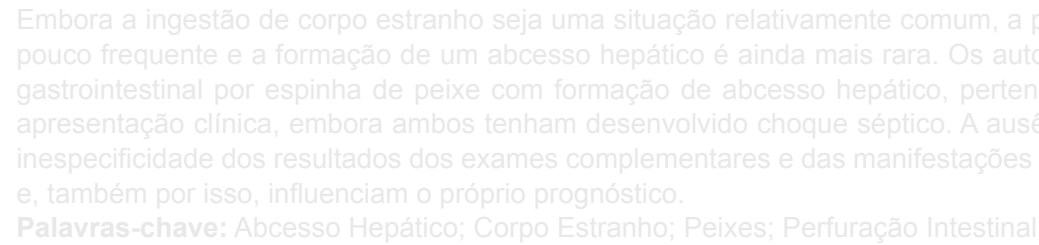

Although foreign body ingestion is a common Case 1

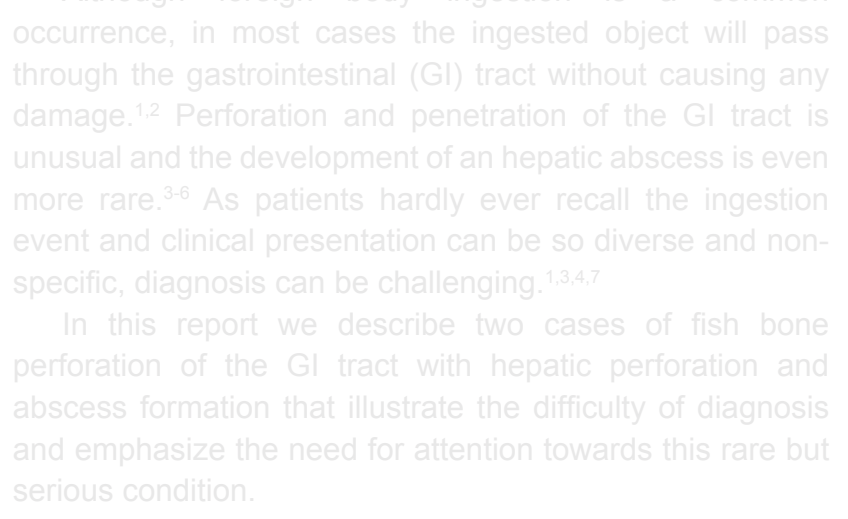

